\title{
Increased phosphorylation of p70 56 kinase is associated with HPVI 6 infection in cervical cancer and esophageal cancer
}

\author{
Y Zhou', Y Pan', S Zhang' ${ }^{2}$ X Shi' ${ }^{2}$ T Ning' and Y Ke*, ${ }^{*, 3,4}$ \\ 'Beijing Institute for Cancer Research, School of Oncology, Peking University No. 52, Fucheng Rd, Hai Dian District, Beijing I00036, PR China; \\ ${ }^{2}$ Department of Surgery, Anyang Cancer Hospital, Anyang City, Henan Province 455000, PR China; ${ }^{3}$ Department of Cell Biology, Health Science Center, \\ Peking University No. 38, Xueyuan Rd, Hai Dian District, Beijing 100083, PR China; ${ }^{4}$ Cancer Research Center, Health Science Center, Peking University \\ No. 38, Xueyuan Rd, Hai Dian District, Beijing 100083, PR China
}

\begin{abstract}
HPVI6 E6 interacts with and degrades tumour suppressor protein TSC2 leading to the phosphorylation of p70 S6 kinase. We studied the association of S6 kinase phosphorylation and HPVI6 infection in cervical cancer and esophageal cancer. Immunohistochemistry was used to assess phosphorylated S6 kinase (Thr 389) and phosphorylated S6 (Ser235/236) in 140 cervical cancer and 16I esophageal cancer specimens. Immunohistochemical staining for pS6 kinase and pS6 was significantly more frequent in the HPVI6-infected cervical cancer specimens than the HPVI6-negative specimens. In contrast, the expression of S6 kinase was similar in both HPVI 6-positive and -negative samples. The phosphorylation of Akt, the key regulator of S6 kinase, was also detected. Our analysis showed that Akt phosphorylation was unaffected by HPVI6 infection. These results together with our previous study suggest that HPVI6 modifies S6 kinase activation via mechanism, which activates S6 kinase downstream of Akt function.
\end{abstract}

British Journal of Cancer (2007) 97, 218-222. doi:10.1038/sj.bjc.6603838 www.bjcancer.com

Published online 10 July 2007

(c) 2007 Cancer Research UK

Keywords: S6 kinase; HPV; Akt; cervical cancer; esophageal cancer

Human papillomavirus (HPV) is a causal agent of cervical cancer and a risk factor for the other epithelial cancer development including esophageal squamous cell carcinomas (zur Hausen, 1999; Bosch et al, 2002; Kari Syrjanen, 2006). Two viral proteins, E6 and E7, are expressed in nearly all cervical cancers and are both necessary and sufficient for immortalisation of primary human keratinocytes in vitro (Hawley-Nelson et al, 1989; Munger et al, 1989; Hudson et al, 1990). The inactivation of tumour suppressor proteins, p53 and Rb by E6 and E7, respectively, plays the major role in HPV-induced tumourigenesis (Dyson et al, 1989; Munger et al, 1989; Scheffner et al, 1990; Werness et al, 1990; Heck et al, 1992). In addition, a growing number of proteins involved in the broad spectrum of cellular functions have been found to be interfered by E6 or E7. We have previously identified tumour suppressor protein TSC2 as the novel target of HPV16 E6. HPV16 E6 interacts with TSC2 and causes the proteasome-mediated degradation of TSC2 resulting in the phosphorylation of S6 kinase even in the absence of insulin (Lu et al, 2004).

Recent studies have revealed that TSC2 plays an important role in the cell growth and proliferation pathway, in which TSC2 forms a functional complex with TSC1 and negatively regulates both growth factor and nutrient-dependent activation of mTOR signalling to its downstream targets S6 kinase and 4EBP1 (Gao et al, 2002; Manning and Cantley, 2003; Saucedo et al, 2003;

*Correspondence: Dr Y Ke;

E-mails: keyang@bjmu.edu.cn and karazhou@163.com

Received 9 January 2007; revised II May 2007; accepted I 4 May 2007; published online 10 July 2007
Stocker et al, 2003; Hay and Sonenberg, 2004). The negative regulatory role of the complex is achieved by TSC2 GTPaseactivating protein activity towards Rheb. Abrogation of TSC2 function by HPV16 E6 may therefore contribute to HPV16-induced proliferation and tumourigenesis.

In this study, we evaluate the phosphorylation of S6 kinase using immunochemical staining in cervical cancer and esophageal cancer tissues and have correlated pS6K staining with HPV16 infection in the patients studied.

\section{MATERIALS AND METHODS}

\section{Patient selection}

One hundred and forty cervical cancers diagnosed between 2000 and 2004, and 161 esophageal cancers diagnosed in 2004 were identified from Anyang Cancer Hospital, China. Tumour material consisted of formalinfixed, paraffin-embedded tumour blocks. Tumours were histologically typed and graded.

\section{Detection of HPV16 infection}

DNA was extracted from the paraffin sections as described (Nindl et al, 2004). For HPV16 DNA detection, E7 was amplified with the forward primer $5^{\prime}$-GATGAAATAGATGGTCCAGC- $3^{\prime}$ and the reverse primer $5^{\prime}$-GCTTTGTACGCACAACCGAAGC-3' as described (Walboomers et al, 1999). In PCR reaction, $\beta$-actin PCR analysis was used to validate the DNA quality. Mouse liver tissue was used in the sample process and PCR analysis to monitor the 
contamination. PCR mixtures contained $10 \mathrm{~mm}$ Tris - $\mathrm{HCl}(\mathrm{pH} 8.3)$, $50 \mathrm{~mm} \mathrm{KCl}, 3.5 \mathrm{~mm} \mathrm{MgCl}_{2}, 0.01 \%$ gelatin, $200 \mathrm{pmol}$ each primer, $4 \mathrm{U}$ Taq DNA polymerase (Promega, Madison, WI, USA) and $100 \mathrm{ng}$ DNA. The cycling conditions were $94^{\circ} \mathrm{C}$ for $40 \mathrm{~s}, 57^{\circ} \mathrm{C}$ for $40 \mathrm{~s}$ and $72^{\circ} \mathrm{C}$ for $40 \mathrm{~s}$ for 40 cycles.

\section{Immunohistochemistry}

Sections were deparaffinised in xylene, washed in graded ethanol and followed in phosphate-buffered saline (PBS). The endogenous peroxidase activity was blocked with PBS containing 3\% hydrogen dioxide. Samples were then pretreated with target retrieval citrate buffer in a microwave for $15 \mathrm{~min}$ and washed three times with PBS.

The nonspecific binding was blocked by incubation in PBS containing $10 \%$ goat serum for $1 \mathrm{~h}$. Slides were stained with IHC- specific phosphorylated Thr 389 S6K antibody (1:500 dilution, Cell Signalling Technologies Inc., Beverly, MA, USA), S6K antibody ( $1: 500$ dilution, Santa Cruz Biotechnology, Santa Cruz, CA, USA), phosphorylated Ser 235/236 S6 antibody (1:100 dilution, Cell Signalling Technologies Inc.) and phosphorylated Ser $473 \mathrm{Akt}$ antibody ( $1: 200$ dilution, Cell Signalling Technologies Inc.) at $4{ }^{\circ} \mathrm{C}$ overnight. The biotinylated secondary antibody and HRP-labelled streptavidin were then added and incubated at $37^{\circ} \mathrm{C}$ for $30 \mathrm{~min}$. The signal was developed in $\mathrm{DAB}-\mathrm{H}_{2} \mathrm{O}_{2}$ solution. The slides were counterstained with hemotaxylin, and then examined by light microscopy. The representative slides stained by different antibodies are shown in Figure 1. The staining was scored on a scale from 0 to IV as follows: 0 , less than $5 \%$ cells were stained; I, $5-25 \%$ cells were stained; II, $25-50 \%$ cells were stained; III, $50-$ $75 \%$ cells were stained; and IV, more than $75 \%$ cells were stained. Scores I-IV were classified as positive, while score 0 was negative.
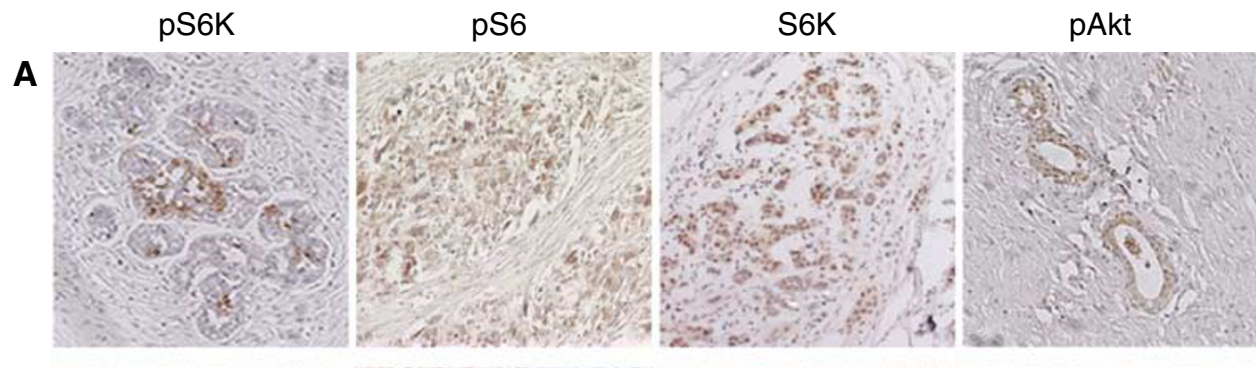

B
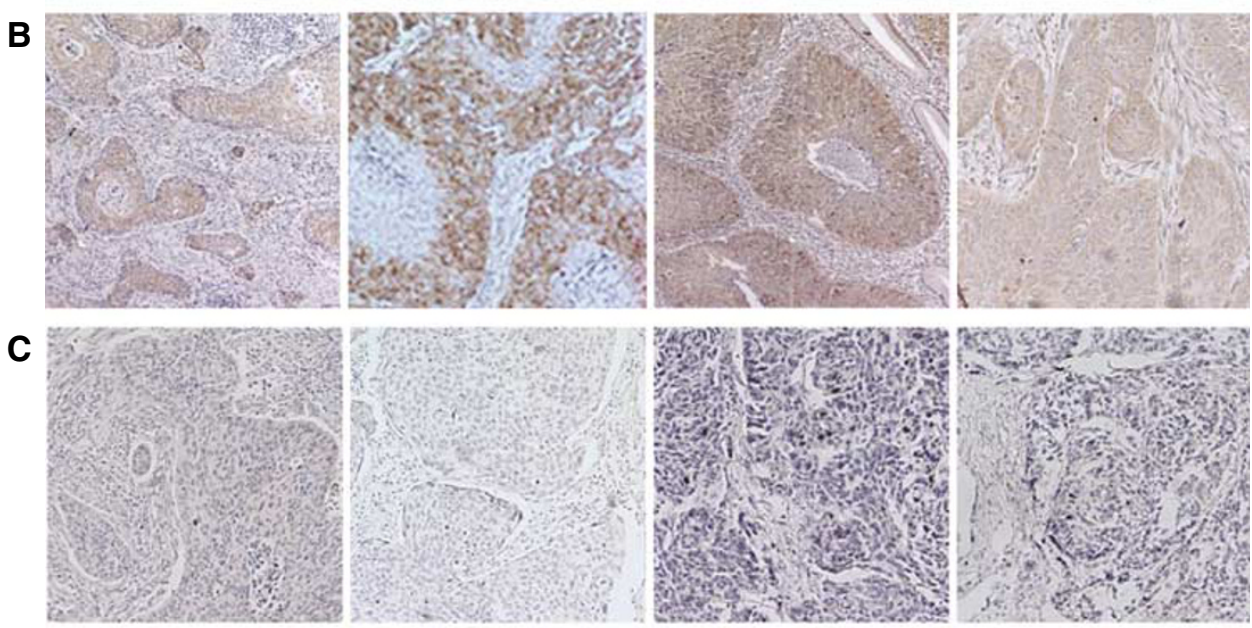

D
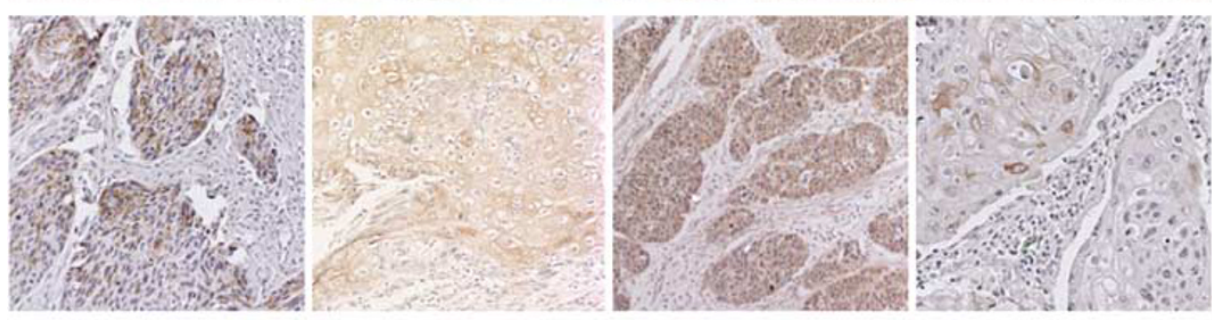

E
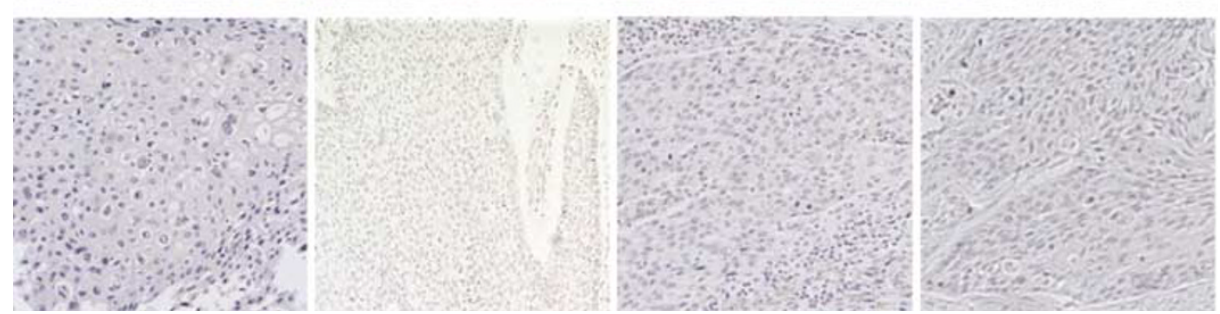

Figure I Immunohistochemistry staining of pS6K, pS6, S6K and pAKT in cervical cancer and esophageal cancer tissues. (A) Positive control of pS6K, pS6, S6K and pAKT in breast cancer (pS6K, pS6, S6K) and prostate cancer (pAKT) tissues. (B) Positive staining of pS6K, pS6, S6K and pAKT in cervical cancer tissues. (C) Negative staining of pS6K, pS6, S6K and pAKT in cervical cancer tissues. (D) Positive staining of pS6K, pS6, S6K and pAKT in esophageal cancer tissues. (E) Negative staining of pS6K, pS6, S6K and pAKT in esophageal cancer tissues. 


\section{Statistical analysis}

The $\chi^{2}$-test was used to investigate the independence of categorical variables. The $P$-value reported was two sided, and values of $P<0.05$ were considered significant. Statistical analysis was performed and statistical calculations were carried out using SAS 8.0.

\section{RESULTS}

Patient characteristics are listed in Table 1 . Since we have found that TSC2 specifically binds to HPV16 E6 and leads to the elevated S6 kinase phosphorylation independent of extra cellular stimuli, to examine if upregulation of active S6 kinase occurs in vivo as a result of HPV16 infection, we determined phosphorylated S6 kinase in cervical cancer and esophageal cancer with or without HPV16 infection. The presence of HPV16 was detected by

Table I Patient characteristics

\begin{tabular}{lcc}
\hline & $\begin{array}{c}\text { Cervical cancer } \\
(\mathbf{N}=\mathbf{I 4 0})\end{array}$ & $\begin{array}{c}\text { ESCC } \\
(\mathbf{N}=\mathbf{I 6} \mathbf{~ I})\end{array}$ \\
\hline $\begin{array}{l}\text { Age (year) } \\
\text { Median (range) }\end{array}$ & $48(21-80)$ & $58(36-77)$ \\
Cell type (\%) & $140(100)$ & $161(100)$ \\
Squamous cell & & \\
HPV status (\%) & $102(72.9)$ & $97(60.2)$ \\
HPV I6+ & $38(27.1)$ & $64(39.8)$ \\
HPV I6- & & 11 \\
Histologic grade & 7 & 102 \\
High-differentiated tumour & 71 & 0 \\
Mild-differentiated tumour & 47 & \\
Low-differentiated tumour & 15 & \\
Undefined cancer & & \\
\hline
\end{tabular}

amplification of HPV16 E7 gene fragment. Among 140 cervical cancer specimens, 102 were HPV16 positive $(72.9 \%)$. In case of esophageal cancer, 97 out of $160(60.2 \%)$ were HPV16 positive.

The phosphorylation of p70 S6 kinase was detected using immunohistochemical stain with antibody specifically against phosphorylated S6 kinase (Thr 389). The degree of immune staining was scored as described in Materials and Methods. In cervical cancer, 43 (42.2\%) HPV16-positive specimens were scored pS6K staining positive I and above, while 3 (7.9\%) HPV16-negative specimens were stained with the same degree $(P=0.000)$. Thirty (29.4\%) HPV16 positives and $1(2.6 \%)$ HPV16 negatives were stained as degree II and above $(P<0.002)$ (Table 2$)$.

In order to confirm the effect of HPV16 on the activation of S6 kinase, we detected the phosphorylation of S6 using anti-phosphoS6 antibody (Ser235/236). Seventy-one (69.6\%) of HPV16-positive specimens were scored degree I and above, and 51 (50\%) of HPV16 positives were degree II and above. In contrast, $12(31.6 \%)$ of HPV16 and $9(23.7 \%)$ of HPV16 negatives were found in the corresponding groups $(P=0.000$ and $P<0.009$, respectively).

In esophageal cancer, 26 (26.8\%) HPV16-positive specimens were pS6K positive with degree I and above staining, while 5 $(7.8 \%)$ of HPV16 negatives were $\mathrm{pS} 6 \mathrm{~K}$ positive with corresponding degree $(P<0.005)$. No significant difference was found between HPV16 positives and negatives with degree II and above pS6Kpositive staining. No significance of pS6 staining at any degree was found between HPV16 positives and negatives (Table 3 ).

In order to exclude the possibility that elevated S6 kinase, phosphorylation was caused by the consequence of increased S6 kinase expression, the specimens were stained with anti-S6 kinase antibody. A 78.4\% of HPV16-positive and 78.9\% of HV16-negative cervical cancers were S6K positive with degree I and above $(P=1.000)$. In esophageal cancers, $61.9 \%$ of HPV16 positives and $56.3 \%$ of HPV16 negatives were S6K positive with degree I and above $(P=0.586)$.

The serine/threonine kinase Akt plays a central role upstream mTOR and S6 kinase in PI3Kinase pathway. The activity of AKT is upregulated in many human cancers leading to S6 kinase

Table 2 Immunohistochemical analysis for p-S6K, p-S6, S6K and p-AKT in cervical cancer tissues

\begin{tabular}{|c|c|c|c|c|c|c|c|c|c|c|c|}
\hline & \multirow[b]{2}{*}{ Group } & \multirow[b]{2}{*}{ Total number } & \multicolumn{9}{|c|}{ IHC score } \\
\hline & & & IV & III & II & $\mathbf{I}$ & 0 & $\geqslant \mathbf{I}(\%)$ & $P$ & $\geqslant$ II (\%) & $P$ \\
\hline & HPVI6- & 38 & I & 0 & 0 & 2 & 35 & $3(7.9)$ & 0.000 & I (2.6) & 0.002 \\
\hline \multirow[t]{2}{*}{ p-S6 } & HPVI6+ & 102 & 18 & 13 & 20 & 20 & 31 & $71(69.6)$ & & $51(50.0)$ & \\
\hline & HPVI6- & 38 & 4 & 3 & 2 & 3 & 26 & $12(31.6)$ & 0.000 & 9 (23.7) & 0.009 \\
\hline S6K & HPVI6+ & 102 & 41 & 11 & 12 & 16 & 22 & $80(78.4)$ & & 64 (62.7) & \\
\hline & HPVI6- & 38 & 0 & 0 & 3 & 2 & 33 & $5(13.2)$ & 0.792 & $3(7.9)$ & 0.965 \\
\hline
\end{tabular}

Table 3 Immunohistochemical analysis for p-S6K, p-S6, S6K and p-AKT in ESCC tissues

IHC score

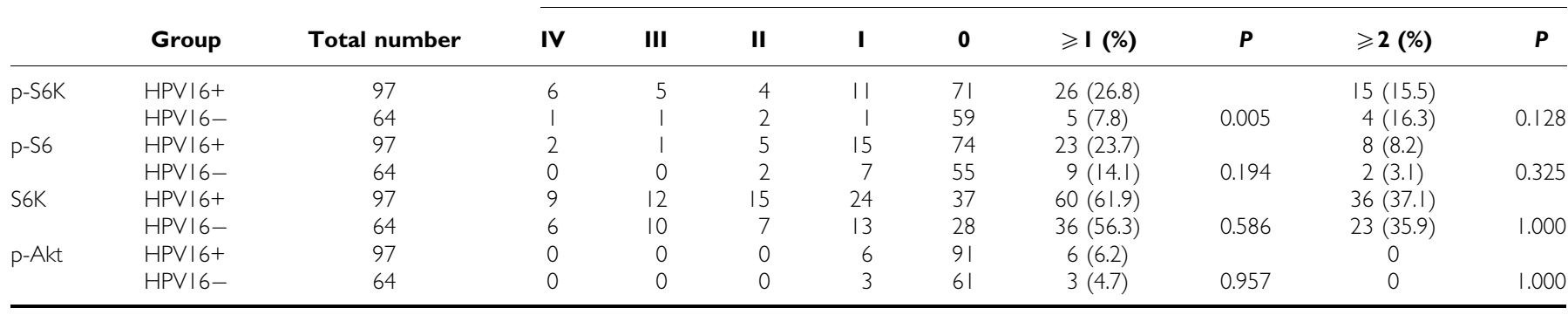


phosphorylation (Brazil et al, 2002; Altomare and Testa, 2005). We next tested the phosphorylation of Akt in HPV16-infected cancer specimens. An antibody specifically against phosphorylated Akt (Ser 473) was used for immunohistochemical stain. In cervical cancer group, 9.8\% HPV16 positives and 13.2\% HPV negatives were pAkt positive with degree I and above $(P=0.792)$. In esophageal cancer group, $6.2 \%$ HPV16 positives and $4.7 \%$ HPV16 negatives were pAkt positive with degree I and above $(P=0.957)$.

The representative examples of pS6K, pS6, S6K and pAkt staining are shown in Figure 1. The brownish signals represent the positive staining. The phosphorylated S6K and S6 were found mainly in the cytoplasm, while S6K was in both cytoplasm and nucleus. The phosphorylated Akt was mainly in the cytoplasm. The positive controls used were a breast cancer specimen for S6K, pS6 and $\mathrm{pS} 6 \mathrm{~K}$, and a prostate cancer specimen for pAkt.

\section{DISCUSSION}

Human papillomaviruses play an etiological role in the development of cervical cancer and several other cancers. Oncogenic HPV E6 and E7 interrupt the host cellular function and therefore take the principal contribution for HPV-induced tumourigenesis. We have previously reported that HPV16 E6 binds to tumour suppressor protein TSC2 and inferences with its suppressor function in insulin-induced cell growth and proliferation-signalling pathway leading to activate S6 kinase downstream of Akt activation ( $\mathrm{Lu}$ et al, 2004). In the present study we demonstrate that S6 kinase phosphorylation was significantly increased in HPV16-infected cervical cancer. In parallel, the phosphorylation of S6 augmented. In HPV16-infected esophageal cancer specimens, the alteration of S6 kinase phosphorylation was not as significant as in cervical cancer specimens. In particular, there was no difference of S6 phosphorylation between HPV16-positive and negative specimens. The discrepancy between cervical cancer and esophageal cancer might be resulted from much lower detectable level of pS6 kinase and pS6 detected in esophageal cancer specimens. Alternatively, the expression of HPV16 E6 may differ in those two group specimens and subsequently affect the activation of S6 kinase differently. We also showed that increased phosphorylation of S6 kinase was resulted from neither elevated S6 kinase expression nor Akt activation in specimens with HPV16 infection.

TSC2 is a key negative regulator of mTOR signalling pathway, in which TSC2 acts as a GTPase-activating protein towards Rheb, thereby inhibiting mTOR. Phosphorylation of TSC2 influences its activity within the complex with TSC1. At least three kinases, Akt, RSK1 and MAPK, are involved in TSC2 phosphorylation (Jozwiak, 2006). Several studies reported that HPV proteins target the components of PI3 kinase and Akt-mediated cell growth pathway. For instance, HPV E6 proteins interact with the membraneassociated guanylate kinase homologues (MAGUKs) MAGI-2 and MAGI-3 for degradation (Thomas et al, 2002). MAGI-2 and MAGI-
3 have been reported to be associated with PTEN (Wu et al, 2000), the other negative regulator of the insulin-induced growth pathway, and enhance the ability of PTEN to suppress Akt activation. The degradation of MAGI-2 and MAGI-3 by E6 may, therefore, result in the abolishment of PTEN function resulting in the activation of Akt-mediated cell growth. In addition, HPV-16 E7 upregulates AKT activity in both Rb-dependent and -independent manner (Westbrook et al, 2002; Pim et al, 2005; Menges et al, 2006). Taken together, these results highlight the importance of interference with the PI3 kinase/Akt-mediated signalling pathway in HPV-induced tumourigenesis. In the current study, we did not observe enhanced Akt phosphorylation in HPV16-positive specimens. This might be caused by that immunohistochemistry is not sensitive enough to detect Akt activation in HPV-infected samples. However, it is also possible that HPV proteins direct target components of this pathway downstream of Akt, or even independent of this pathway in certain condition. Therefore, the activation of Akt might remain unchanged. For instance, our previous study showed that expression of HPV16 E6 promoted the degradation of TSC2 and phosphorylation of S6 kinase and S6 in the absence of insulin stimulation, while phosphorlation of Akt was unchanged in the presence and absence of HPV16 E6 (Lu et al, 2004). The results presented here support our previous study.

In addition, HPV proteins also activate MAPK pathway. HPV16 E6 increases the phosphorylation of MAPK and MER (Chakrabarti et al, 2004). HPV16 E5 promotes the activation of EGFR and thus enhances the RAS/RAF/MAPK signalling cascade (Kim et al, 2006). Further investigation on why and how HPV oncoproteins preferentially target the different components in this pathway will be of great help for understanding HPV-induced tumourigenesis.

Recent studies also found that amplification of the PIK3CA gene accompanied by serine 473 phosphorylation of AKT is common in cervical cancers and it seems to be an independent event to HPV infection (Ma et al, 2000; Zhang et al, 2002; Bertelsen et al, 2006). Under our current condition, only $9.8 \%$ of cervical cancers and $6.2 \%$ of esophageal cancers were pAkt positive. This discrepancy is unlikely due to a technical issue since we have repeated the detection using antibodies from different batches and a positive control from the manufacture was always used to monitor the sensitivity. One possible explanation is that PIK3CA gene amplification is corresponding to the progression of disease. In our analysis, tumours were not classified by stages. In addition, Kim et al (2006) reported that Akt phosphorylation contributes to radioresistance in cervical cancer. A more detailed analysis is required to define the Akt phosphorylation-related disease stage and progression.

\section{ACKNOWLEDGEMENTS}

This work was supported by Natural Science Foundation of China 30430710 to Yang Ke.

\section{REFERENCES}

Altomare DA, Testa JR (2005) Perturbations of the AKT signaling pathway in human cancer. Oncogene 24: 7455-7464

Bertelsen BI, Steine SJ, Sandvei R, Molven A, Laerum OD (2006) Molecular analysis of the PI3K-AKT pathway in uterine cervical neoplasia: frequent PIK3CA amplification and AKT phosphorylation. Int $J$ Cancer 118: $1877-1883$

Bosch FX, Lorincz A, Munoz N, Meijer CJLM, Shah KV (2002) The causal relation between human papillomavirus and cervical cancer. J Clin Pathol 55: 244-265

Brazil DP, Park J, Hemmings BA (2002) PKB binding proteins. Getting in on the Akt. Cell 111: 293-303
Chakrabarti O, Veeraraghavalu K, Tergaonkar V, Liu Y, Androphy EJ, Stanley MA, Krishna S (2004) Human papillomavirus type 16E6 amino acid 83 variants enhance E6-mediated MAPK signaling and differentially regulate tumorigenesis by notch signaling and oncogenic Ras. J Virol 78: $5934-5945$

Dyson N, Howley PM, Munger K, Harlow E (1989) The human papilloma virus-16 E7 oncoprotein is able to bind to the retinoblastoma gene product. Science 243: 934-937

Gao X, Zhang Y, Arrazola P, Hino O, Kobayashi T, Yeung RS, Ru B, Pan D (2002) Tsc tumour suppressor proteins antagonize amino-acid-TOR signaling. Nat Cell Biol 4: 699-704 
Hawley-Nelson P, Vousden KH, Hubbert NL, Lowy DR, Schiller JT (1989) HPV16 E6 and E7 proteins cooperate to immortalize human foreskin keratinocytes. EMBO J 8: $3905-3910$

Hay N, Sonenberg N (2004) Upstream and downstream of mTOR. Genes Dev 18: 1926 - 1945

Heck DV, Yee CL, Howley PM, Munger K (1992) Efficiency of binding the retinoblastoma protein correlates with the transforming capacity of the E7 oncoproteins of the human papillomaviruses. Proc Natl Acad Sci USA 89: $4442-4446$

Hudson JB, Bedell MA, McCance DJ, Laiminis LA (1990) Immortalization and altered differentiation of human keratinocytes in vitro by the E6 and E7 open reading frames of human papillomavirus type 18. J Virol 64: $519-526$

Jozwiak J (2006) Hamartin and tuberin: working together for tumor suppression. Int J Cancer 118: 1 -5

Kari Syrjanen M (2006) HPV and oesophageal carcinoma. In: Papillomavirus Research from Natural History to Vaccines and Beyond, Campo S (ed). pp 229-253. Caister Academic: Great Britain

Kim SH, Juhnn YS, Kang S, Park SW, Sung MW, Bang YJ, Song YS (2006) Human papillomavirus 16 E5 up-regulates the expression of vascular endothelial growth factor through the activation of epidermal growth factor receptor, MEK/ ERK1, 2 and PI3K/Akt. Cell Mol Life Sci 63: $930-938$

Kim TJ, Lee JW, Song SY, Choi JJ, Choi CH, Kim BG, Lee JH, Bae DS (2006) Increased expression of pAKT is associated with radiation resistance in cervical cancer. $B r$ J Cancer 94: $1678-1682$

Lu Z, Hu X, Li Y, Zheng L, Zhou Y, Jiang H, Ning T, Basang Z, Zhang C, $\mathrm{Ke} \mathrm{Y}$ (2004) Human papillomavirus 16 E6 oncoprotein interferences with insulin signaling pathway by binding to tuberin. J Biol Chem 279: $35664-35670$

Ma YY, Wei SJ, Lin YC, Lung JC, Chang TC, Whang-Peng J, Liu JM, Yang DM, Yang WK, Shen CY (2000) PIK3CA as an oncogene in cervical cancer. Oncogene 19: 2739-2744

Manning BD, Cantley LC (2003) Rheb fills a GAP between TSC and TOR Trends Biochem Sci 28: $573-576$

Menges CW, Baglia LA, Lapoint R, McCance DJ (2006) Human papillomavirus type $16 \mathrm{E} 7$ up-regulates $\mathrm{AKT}$ activity through the retinoblastoma protein. Cancer Res 66: 5555 - 5559

Munger K, Werness BA, Dyson N, Phelps WC, Harlow E, Howley PM (1989) Complex formation of human papillomavirus E7 proteins with the retinoblastoma tumor suppressor gene product. EMBO J 8: 4099-4105
Nindl I, Meyer T, Schmook T, Ulrich C, Ridder R, Audring H, Sterry W, Stockfleth E (2004) Human papillomavirus and overexpression of P16INK4a in nonmelanoma skin cancer. Dermatol Surg 30: 409-414

Pim D, Massimi P, Dilworth SM, Banks L (2005) Activation of the protein kinase B pathway by the HPV-16 E7 oncoprotein occurs through a mechanism involving interaction with PP2A. Oncogene 24: $7830-7838$

Saucedo LJ, Gao X, Chiarelli DA, Li L, Pan D, Edgar BA (2003) Rheb promotes cell growth as a component of the insulin/TOR signalling network. Nat Cell Biol 5: 566-571

Scheffner M, Werness BA, Huibregtse JM, Levine AJ, Howley PM (1990) The E6 oncoprotein encoded by human papillomavirus types 16 and 18 promotes the degradation of p53. Cell 63: 1129-1136

Stocker H, Radimerski T, Schindelholz B, Wittwer F, Belawat P, Daram P, Breuer S, Thomas G, Hafen E (2003) Rheb is an essential regulator of S6K in controlling cell growth in Drosophila. Nat Cell Biol 5: $559-565$

Thomas M, Laura R, Hepner K, Guccione E, Sawyers C, Lasky L, Banks L (2002) Oncogenic human papillomavirus E6 proteins target the MAGI-2 and MAGI-3 proteins for degradation. Oncogene 21: $5088-5096$

Walboomers JM, Jacobs MV, Manos MM, Bosch FX, Kummer JA, Shah KV, Snijders PJ, Peto J, Meijer CJ, Munoz N (1999) Human papillomavirus is a necessary cause of invasive cervical cancer worldwide. J Pathol 189: $12-19$

Werness BA, Levine AJ, Howley PM (1990) Association of human papillomavirus types 16 and 18 E6 proteins with p53. Science 248: 76-79

Westbrook TF, Nguyen DX, Thrash BR, McCance DJ (2002) E7 abolishes raf-induced arrest via mislocalization of p21(Cip1). Mol Cell Biol 22: $7041-7052$

Wu X, Hepner K, Castelino-Prabhu S, Do D, Kaye MB, Yuan XJ, Wood J, Ross C, Sawyers CL, Whang YE (2000) Evidence for regulation of the PTEN tumor suppressor by a membrane-localized multi-PDZ domain containing scaffold protein MAGI-2. Proc Natl Acad Sci USA 97: $4233-4238$

Zhang A, Maner S, Betz R, Angstrom T, Stendahl U, Bergman F, Zetterberg A, Wallin KL (2002) Genetic alterations in cervical carcinomas: frequent low-level amplifications of oncogenes are associated with human papillomavirus infection. Int J Cancer 101: 427 - 433

zur Hausen H (1999) Papillomaviruses in human cancers. Proc Assoc Am Physicians 111: $581-587$ 